\title{
Supporting Information for: \\ Generation of Nanoparticles from Friction between Railway Brake Disks and Pads
}

Hyeong-Gyu Namgung ${ }^{1}$, Jong-Bum Kim ${ }^{2,3}$, Sang-Hee Woo ${ }^{2}$, Sechan Park ${ }^{1,5}$, Minhae Kim ${ }^{1,5}$ Min-Soo Kim ${ }^{4}$, Gwi-Nam Bae ${ }^{2}$, Duckshin Park ${ }^{1}$, Soon-Bark Kwon ${ }^{1,5^{*}}$

${ }^{1}$ Transportation Environmental Research Team, Korea Railroad Research Institute (KRRI), Uiwang-si, Korea 16105

${ }^{2}$ Center for Environment, Health and Welfare Research, Korea Institute of Science and Technology (KIST), Seoul, Korea 02792

${ }^{3}$ Green School (Graduate School of Energy, Environment Policy \& Technology), Korea University, Seoul, Korea 02841

${ }^{4}$ Metropolitan Transportation Research Center, Korea Railroad Research Institute (KRRI), Uiwang-si, Korea 16105

${ }^{5}$ Railway System Engineering, University of Science and Technology (UST), Uiwang-si, Korea 16105

\section{EXPERIMENTAL METHODS}

Method S1: Description of measurement methods, particle sampling loss and uncertainty of the FMPS. (Page S2)

Method S2: Experimental conditions and background level treatment (Page S3)

\section{FIGURES}

Figure S1. TEM Images of collected airborne wear particles (Page S4)

REFERENCES (Page S5)

*Corresponding author contact information: Korea Railroad Research Institute (KRRI), Uiwang-si, Korea 16105. Email sbkwon@krri.re.kr 


\section{EXPERIMENTAL METHODS}

Method S1 Description of measurement methods, particle sampling loss and uncertainty of the FMPS.

We used the 4-way flow splitter which has a maximum flow rate of $30 \mathrm{lpm}$. The flow rate of each device was $10 \mathrm{lpm}$ for FMPS, $1.2 \mathrm{lpm}$ for OPC, $5 \mathrm{lpm}$ for APS, and $2 \mathrm{lpm}$ for the air sampler for TEM analysis, and $2.5 \mathrm{lpm}$ for the air sampler for SEM analysis (total sampling flow rate was $20.7 \mathrm{lpm}$ ). A transport loss of particles during the sampling mainly due to diffusional deposition was calculated: transport loss of $10 \mathrm{~nm}$-sized particles was less than $7 \%$ at the lowest sampling flow rate (i.e., 2 lpm, air sampler for TEM analysis) and that of $500 \mathrm{~nm}$-sized particles was less than $1 \%$ in the worst case. ${ }^{1}$ The first three devices had different diameter measurement ranges: APS was able to measure aerodynamic particles with diameters ranging from 0.5 to $20 \mu \mathrm{m}$ at one-second intervals; OPC was able to measure those particles ranging from 0.25 to $32 \mu \mathrm{m}$ at six-second intervals; and FMPS was able to measure those particles ranging from 5.6 to $560 \mathrm{~nm}$ at one-second intervals, offering 32 channels of resolution. The measurement uncertainty of the instrument was not reported by the manufacturer, however, several studies reported comparative results with a scanning mobility particle sizer (SMPS) which is a known reference instrument that measures 5 700 nm-sized particles at 1 2 min time resolution. ${ }^{2-4}$ In addition, disk temperature was concurrently measured near the sizers and the counter with a thermometer (Type $\mathrm{K}$ thermocouple, $\pm 0.4 \%$ uncertainty) built in the disk and thermographic camera ( 870 pro, Testo) mounted on the side of the dynamometer. Air samplers were used to collect airborne wear particles released during braking. The air sampler (Nanoparticle Collector, HTC) for TEM analysis was equipped with TEM grid (Quantifol, Electron Microscopic Science) and the air sampler (Gilian air sampler, Sensidyne) for SEM analysis was equipped with a membrane filter (pore size $0.1 \mu \mathrm{m}, 47 \mathrm{~mm}$ diameter, Millipore). Collected samples were analyzed by a transmission electron microscope (TEM; F20 G2, Tecnai) and a field emission-scanning electron microscope (FE-SEM; S$5000 \mathrm{H}$, Hitachi) at a separate analysis laboratory after the test. An elemental analysis was conducted with an energy-dispersive X-ray spectrometer (EDX). This study looked only at the results from the FMPS, SEM and TEM in figuring out the change properties of nanoparticle size distribution. 
Method S2 Experimental conditions and background level treatment

The experimental conditions used in this study were set up based on the “International Union of Railways (UIC) code 541-3". UIC code 541-3 contains the general conditions for disk brakes and their indicating equipment for railway trains. ${ }^{5}$ Before commencing the test for each case, the ventilation device was run long enough to remove pollutants from the area around the dynamometer. A background particle concentration was measured before the first testing round as a criterion of background level. Subsequent rounds of testing were conducted only when the background level approached to criteria. In addition, we disregarded the background level concentration for analysis of pure brake wear particles only. When the targeted test speed had been achieved, the brake was applied until the disk stopped rotating, at which point the first round of testing ended. 


\section{FIGURES}

Figure S1 Results of a TEM image analysis of the samples of airborne particles generated during braking in the $\mathrm{S} 4$ condition (at three different brake forces).

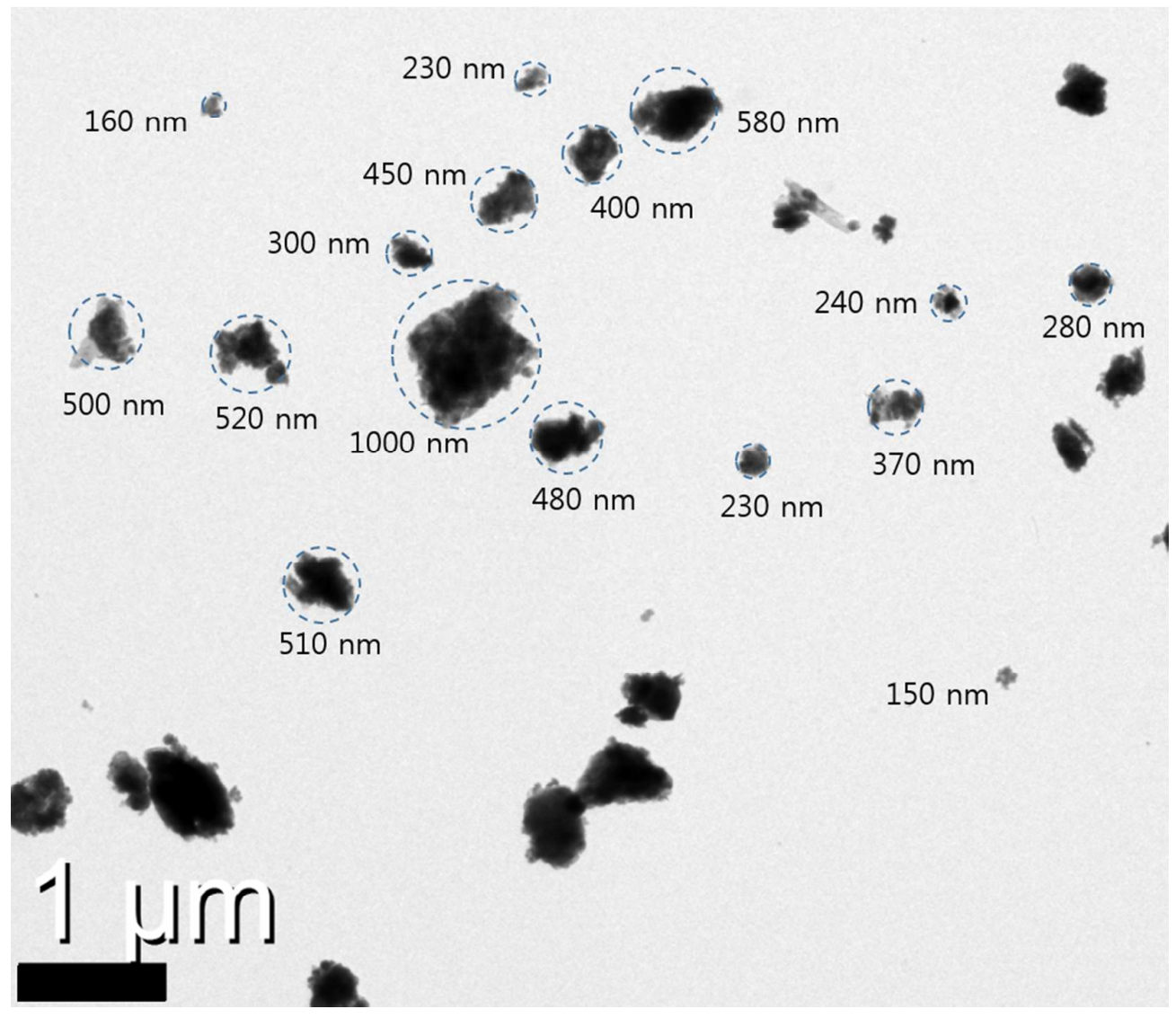

Figure S1. TEM Images of collected airborne wear particles. 


\section{REFERENCES}

1. Hinds, W. C., Aerosol Technology, 2nd ed.; John Wiley \& Sons, Inc.: USA, 1982.

2. Lee, B. P.; Li, Y. J.; Flagan, R. C.; Lo, C.; Chan, C. K. Sizing characterization of the fast-mobility particle size (FMPS) aginst SMPS and HR-ToF-AMS. Aerosol Sci. Tech. 2013, 47, 1030-1037.

3. Jeong, C. H.; Evans, G. J. Inter-comparison of a fast mobility particle sizer and a scanning mobility particle sizer incorporating an ultrafine water-based condensation particle counter. Aerosol Sci. Tech. 2009, 43, 364-373.

4. Zimmerman, N.; Pollitt, K. J. G.; Jeong, C. H.; Wang, J. M.; Jung, T. Cooper, J. M.; Wallace, J. S.; Evans, G. J. Comparison of three nanoparticle sizing instruments: The influence of particle morphology. Atmos. Environ. 2014, 86, 140-147.

5. UIC code 541-3, Brakes-Disk brakes and their application - General conditions for the approval of brake pads, International Union of Railways, $\sigma^{\text {th }}$ edition, November 2006. 